\title{
Use of webcams as tools for alignment and supervision of a Thomson scattering system in the near infrared
}

\author{
Y. Andrèbe*, R. Behn, B.P. Duval, P. Etienne, A. Pitzschke \\ Ecole Polytechnique Fédérale de Lausanne (EPFL), Centre de Recherches en Physique des Plasmas, Association Euratom-Confédération Suisse, Station 13, 1015 Lausanne, Switzerland
}

\section{A R T I C L E I N F O}

\section{Article history:}

Available online $\mathrm{xxx}$

\section{Keywords:}

Webcam

Near infrared

Alignment

Nd:YAG laser

Thomson scattering

\begin{abstract}
A B S T R A C T
The alignment stability is a major concern for Thomson scattering systems. Even small angular deviations of the laser beams crossing the plasma lead to a loss of the calibration resulting in unreliable measurements of the electron density profile. For the TCV (Tokamak à Configuration Variable) installation, the beam paths from the laser output to the vacuum chamber are $\sim 25 \mathrm{~m}$ long and include several optical components. In order to monitor the alignment on a regular basis, a set of 9 cameras has been installed at several locations along the beam path. They view the actual laser beam pattern by recording the scattered light from an intercepting optical surface (mirror or window) together with the position of markers used for reference. Small "webcams" are used for this purpose; they feature adequate intensity response at the laser wavelength of $1.06 \mu \mathrm{m}$, are compact, cheap and several units may be connected to a server PC simultaneously. The real-time images from all the cameras are accessible from a Web browser. This installation has proven to be extremely useful in the early detection of alignment problems and to assist the alignment procedure [1].
\end{abstract}

(c) 2011 ASSOCIATION EURATOM, Confederation Suisse, EPFL CRPP. Published by Elsevier B.V. All rights reserved.

\section{The Thomson scattering diagnostic}

The Thomson scattering system is the main diagnostic for the measurement of the spatial profiles of the electron temperature and density on TCV.

Three Nd:YAG lasers (Table 1) are installed in a laser room outside the Tokamak area. This allows permanent access to the laser system, even during TCV experiments, and permits operation in a safe, dust-free and temperature stabilized environment.

The laser beam paths from the lasers to the TCV vacuum chamber are about $25 \mathrm{~m}$ long. A beam expander (Galilean telescope, expansion ratio of 2) in front of the lasers increases the beam diameters to $20 \mathrm{~mm}$. This reduces the divergence and energy density of the laser beam on the optical components.

The beams are reflected by 12 folding mirrors before being focused into the torus by a lens assembly. The focusing system has a long focal length $(4.3 \mathrm{~m})$ to obtain an extended observation range with the focussed beam varying only weakly ( $2 \mathrm{~mm}$ at the vessel midplane to $4 \mathrm{~mm}$ at the outermost observation points).

The Thomson scattering light from the vertical beams is collected by 3 wide angle camera lenses installed into horizontal ports and imaged onto optical fibre bundles. The $3.3 \mathrm{~mm}$ width of the

\footnotetext{
* Corresponding author. Tel.: +41216934339.

E-mail address: yanis.andrebe@epfl.ch (Y. Andrèbe).
}

observation volume, depends on the fibres' diameter $(1 \mathrm{~mm})$ and the camera lens magnification (0.3). The optical fibres transfer the collected light to polychromators equipped with 3 or 4 interference filters and Si-avalanche photodiodes which finally measure the scattered light intensity. The system is absolutely calibrated using Raman scattering from nitrogen specially introduced into the TCV vessel.

This Thomson scattering system is capable of measuring electron temperatures over a range from $50 \mathrm{eV}$ to $20 \mathrm{keV}$ and electron densities down to $3 \times 10^{18} \mathrm{~m}^{-3}$ for 35 spatial channels. This system has been used since 1994 as a key diagnostic for heating, confinement, ECCD and stability studies at TCV.

\section{Alignment monitoring}

Alignment stability is a major concern of the Thomson scattering system. Even small angular deviations of the laser beams lead to loss of calibration and result in unreliable measurements of the electron density profile. Although the requirements for passive stability were considered in the design, occasional perturbations and slow drifts could not be excluded.

Alignment of 3 high-power laser beams along a complex optical path is a tedious and often time consuming task. In general it requires access to the beam paths, special tools for visualisation of the beam position and intrusive safety precautions. Various solutions have been implemented on other Thomson 
Table 1

Nd:YAG laser features.

\begin{tabular}{ll}
\hline Wavelength & $1064 \mathrm{~nm}$ \\
Frequency & $20 \mathrm{~Hz}$ \\
Energy & $1.8 \mathrm{~J}$ \\
Pulse width & $8-12 \mathrm{~ns}$ \\
Beam diameter & $10 \mathrm{~mm}$ \\
Divergence & $<0.5 \mathrm{mrad}$ \\
Spatial mode profile (far field) & Multimode \\
Polarization & Linear \\
\hline
\end{tabular}

Scattering diagnostic to simplify or automated the alignment procedure [2-4]. On TCV this alignment has to be readjusted 1 or 2 times per month on average. So we have decided to equip this diagnostic with a beams monitoring system, able to detect any misalignment during the warm up period of 4 before each plasma shot and enable to simplify and accelerate the alignment procedure[1]. To this end nine cameras were fixed along the beam path (Fig. 1).

\section{The webcams alternative}

Various types of cameras for scientific applications and sensitive in the near-infrared are available commercially. Dedicated systems for quantitative measurements of laser beam profiles exist, but still are expensive as they offer features that are not required for the application envisaged. But on TCV a large number of cameras are required due to the complexity of the optical path and that some cameras may need to be replaced in case of damage by direct exposure to the laser beams (misalignment or during the alignment procedure).

Thereby commercial USB webcams were selected and two models tested and installed into the optical path:

- A webcam equipped with a 1/4 in. CMOS VGA sensor, $640 \times 480$ pixels, $\sim 20 €$.

- A webcam equipped with a 1/4 in. CCD VGA sensor (Sony ICX098BQ), $640 \times 480$ pixels, $\sim 75 €$.

They permit to visualize the beam pattern of the high-power IR laser by recording scattered light from an optical surface of the beam path (mirror or window). The Nd:YAG lasers used for the Thomson scattering system deliver $1.8 \mathrm{~J} /$ pulse and are strongly attenuated by a highly reflecting mirror used to intercept the beam in the laser room during the warm up period before a plasma shot. This fraction of $0.23 \%$ (i.e. $4 \mathrm{~mJ} /$ pulse) was found sufficient for the webcams. The mirror is sufficiently thin to cause only negligible beam displacement, such that alignment can be performed at low laser power offering a welcome safety advantage during the alignment process.

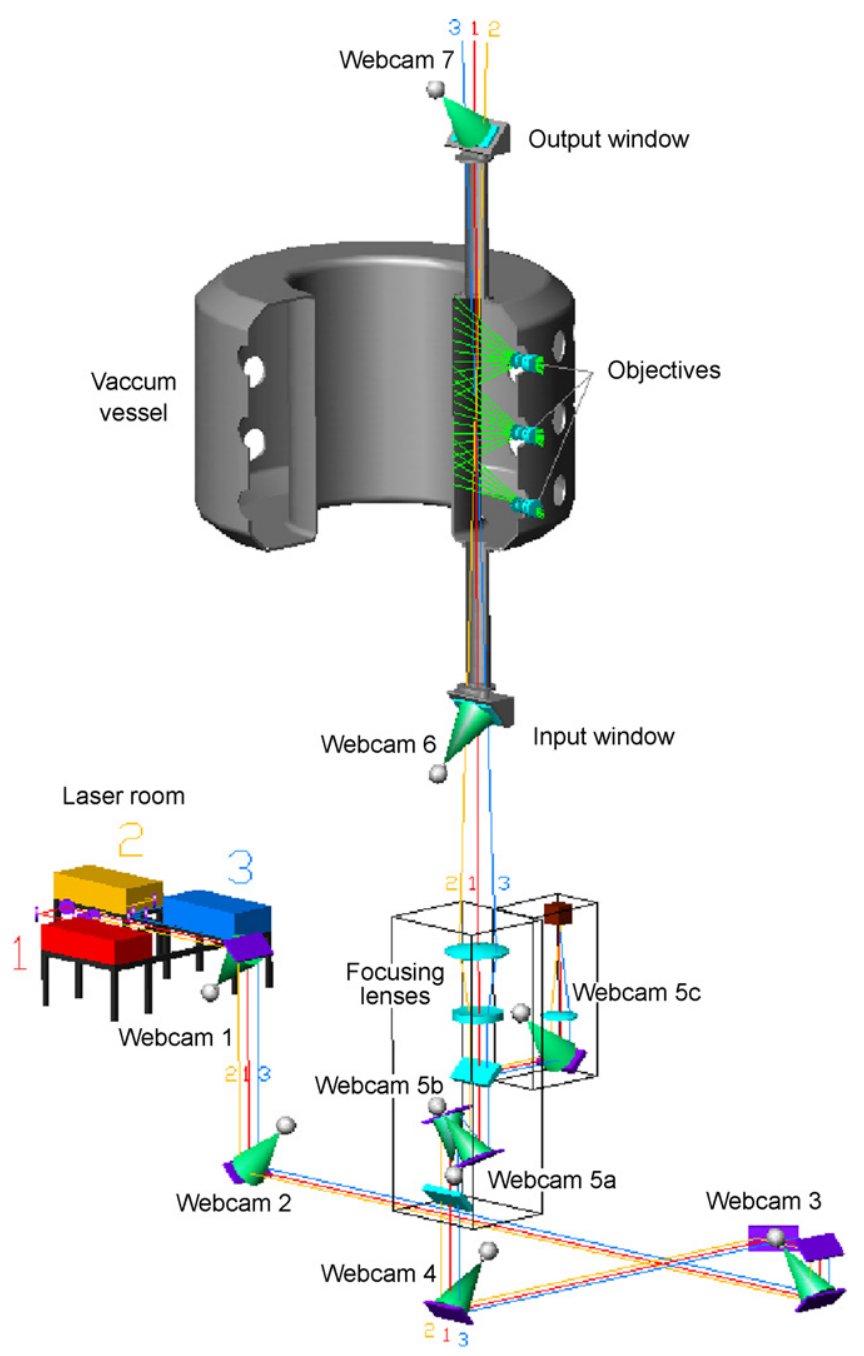

Fig. 1. The nine cameras on the laser beam path.

Both the CCD (Charge-Coupled Devices) and CMOS (Complementary Metal-Oxide Semiconductor) are sensitive at $1064 \mathrm{~nm}$ with similar spectral response since both use similar silicon photodiode elements.

The webcams were designed for colour imaging and are equipped with a colour filter. Fortunately, these RGB filters (Bayer filter) retain some transmission in the near infrared (Fig. 2).

To remove these wavelengths, manufacturers insert an IR blocking filter. When working at low laser energy case $(4 \mathrm{~mJ}$ per pulse in a $20 \mathrm{~mm}$ diameter beam diffused on a mirror surface), it was

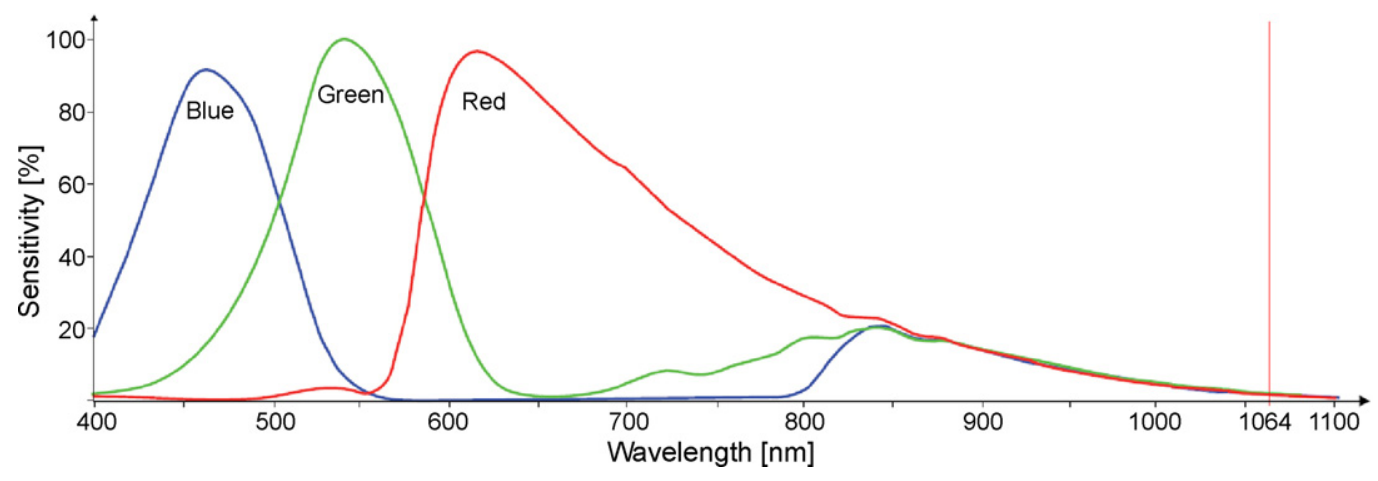

Fig. 2. Sony ICX098BQ datasheet-spectral sensitivity of the CCD sensor. 


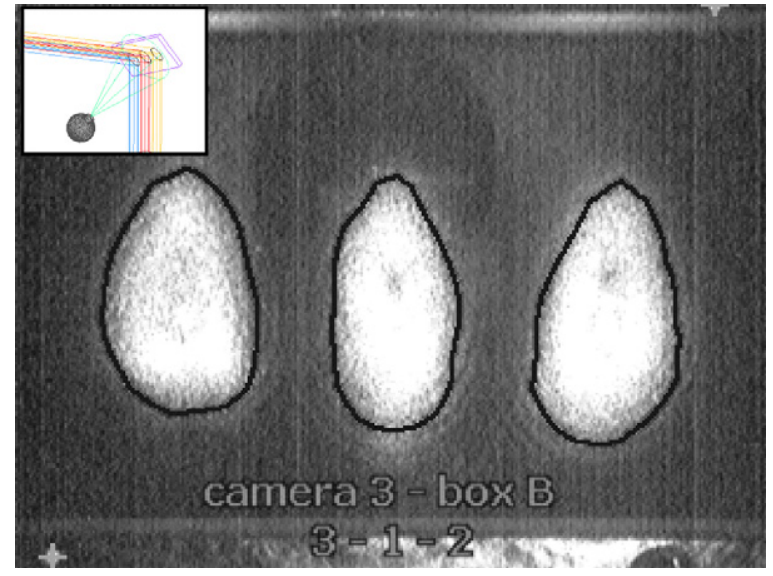

Fig. 3. Laser beam pattern on a mirror surface at $45^{\circ}$ inside theirs reference marks. The resolution is limited to $320 \times 240$ pixels.

necessary to remove this filter. This filter was either a coating on a flat window located between the lens and the sensor, or a coating directly applied on the lens. In the first case it was simply removed, but in the second a better solution was to replace the lens. Microvideo lenses threaded for M12 $\times 0.5 \mathrm{~mm}$ (compatible for webcam) without IR blocking filter are easily purchased.

The image quality of the webcams (vignetting, aberrations, etc.) is not important since we are only interested in the laser beam pattern together with a marker or target point (Fig. 3).

Both webcams have a resolution of $640 \times 480$ pixels but were limited to $320 \times 240$ pixels to allow the computer to simultaneously display all nine images. This resolution is sufficient provided that the image size of the laser beam is large enough on the sensor.

A webcam could not be triggered by the laser pulse so a sufficiently long exposure time was employed. The webcam driver allows exposure times up to $200 \mathrm{~ms}$, integrating 4-5 laser pulses. With an exposure time from $50 \mathrm{~ms}$ to $200 \mathrm{~ms}$ at least one pulse will be acquired whereas below $50 \mathrm{~ms}$ the webcam may not record any pulse due to lack of synchronization. Since the cameras are installed inside a closed beam duct (enclosure of the laser beam path), there is no risk of saturation due to ambient light when the webcam uses a long exposure time.

A major advantage of the webcam is its size. It is very compact and can be integrated without major difficulty into the optical path, close to the main optical components (Fig. 4).

The cabling of the webcams is simplified since no trigger is required and the USB cable carries both the power and the acquired image. To power all 9 cameras, a self-powered usb hub was installed and for cable lengths in excess of $5 \mathrm{~m}$, an active usb extension cable was installed. Although, theoretically, up to 5 extensions may be

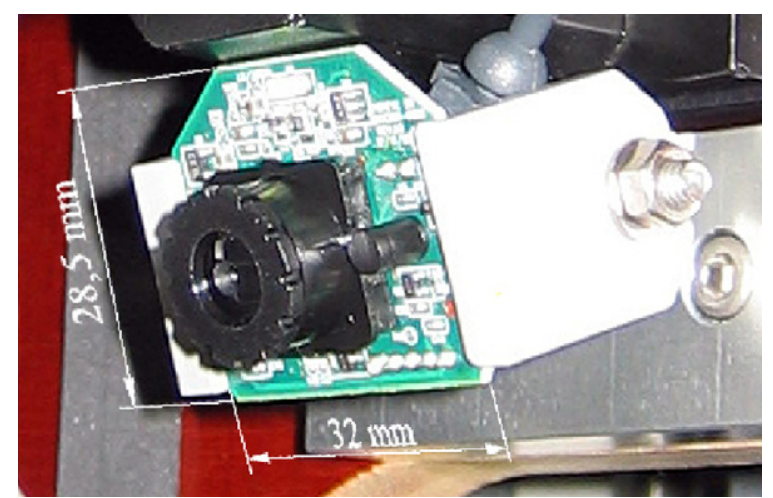

Fig. 4. Webcam stripped of its shell.

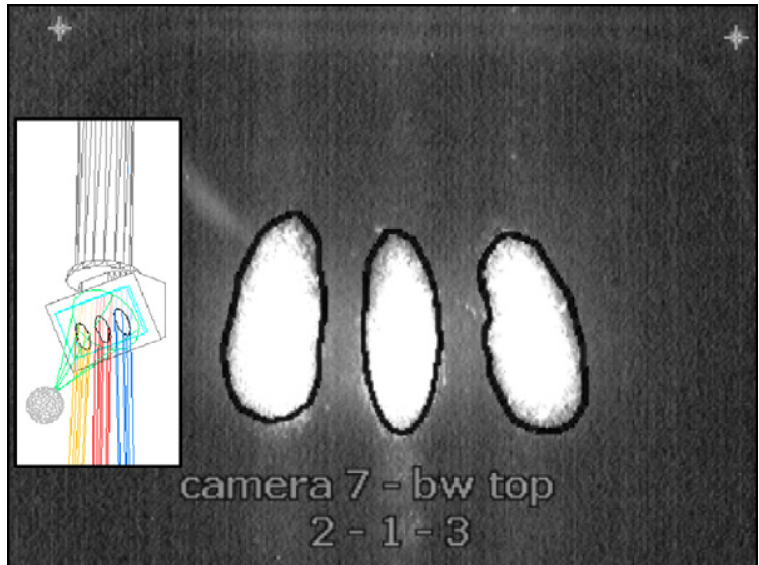

Fig. 5. Laser beam pattern at the output of a window at Brewster angle (56 ) inside theirs reference marks. The two crosses on the top corners mark the position of the camera relative to a fix point in the scenery.

used ( $25 \mathrm{~m}$ ), only three performed reliably in practice so for longer distances, fibre optical USB extensions may be used.

All the webcams are linked to a computer that acts as a server. Real-time images from all the cameras are accessible using a Web browser. The possibility to visualize these images from any place using a laptop with a Wi-Fi connection is very convenient. Software permits overlays on the video streams. Thus, after an initial alignment, the position and size of the 3 beams are marked by software. The marker remains valid as long as the cameras do not move. Additional markers may also be added to fix elements of the scenery as references (Fig. 5).
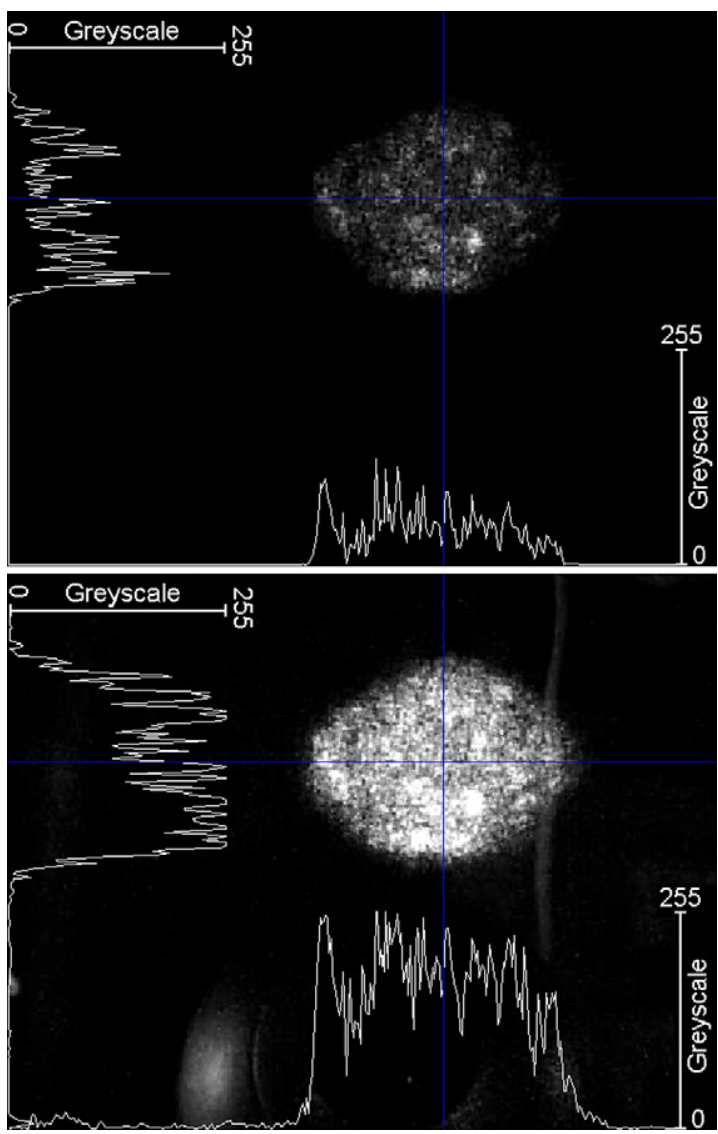

Fig. 6. Webcam (CMOS sensor) perpendicular to the mirror surface, $5 \mathrm{~mJ} /$ pulse at $20 \mathrm{~Hz}$, beam diameter $10 \mathrm{~mm}$. On the top: exposure time $40 \mathrm{~ms}$ ( 1 pulse). On the bottom: exposure time $200 \mathrm{~ms}$ ( 4 or 5 pulses). 


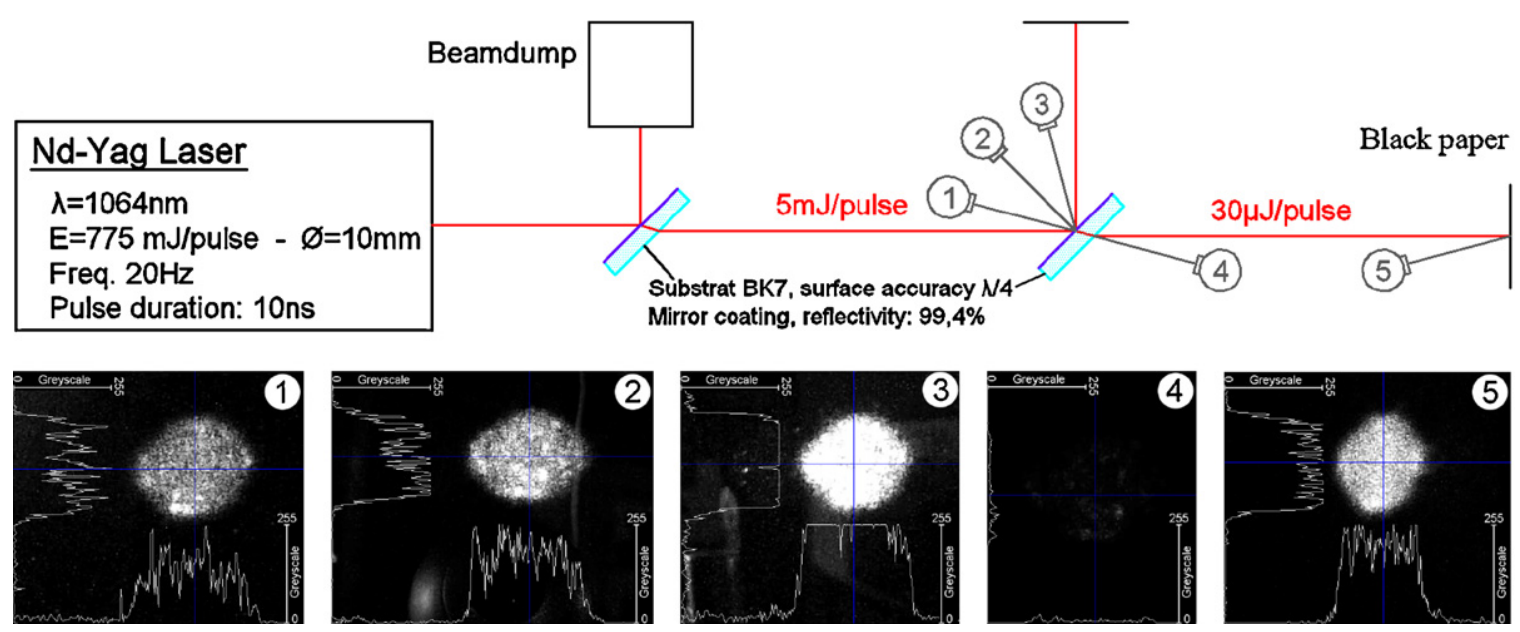

Fig. 7. Change of the position of a webcam (CMOS) relative to a mirror, exposure time $200 \mathrm{~ms}$, measurements in the darkness.

From simultaneous recordings of many webcams, a misalignment in the laser beam path is easily detected and adjustments can be made rapidly using the remotely controllable mirrors. Changes in the beam diameter, which could lead to dangerous increase in the local power densities on optical components, are also readily identified.

\section{Laboratory measurements}

The response of webcams to different illumination conditions was quantified before their installation.

Along the optical path, the laser beams encounters various optical surfaces: mirrors, windows at Brewster angle and lenses with anti-reflection coating. Although high-quality optical components are used, there is still sufficient scatter off the small-scale imperfections (surface roughness) to be detected by the webcams. The amount of scattered radiation depends on the surface quality of the substrates (our substrates are polished to $\lambda / 4$ ), the cleanliness of the surfaces and the energy density of the laser beam.

A webcam observing a mirror surface show quite clearly this diffusion. Two parameters can be modified to adjust the level of diffusion visible by the webcam: the exposure time (Fig. 6) and the angle of the camera relative to the specular reflection. The signal increases as the camera is positioned closer to the specular reflection angle (Fig. 7).
On windows and lenses, the quantity of scattered light captured by the webcam increases if placed on the exit rather than the entrance side of the optical element.

\section{Conclusion}

A set of simple webcams with CMOS or CCD Si-sensors have been used to monitor and align the laser beam path in a Thomson scattering system. The cameras are sensitive enough to detect the small fraction of scattered intensity off intersecting optical surfaces like mirrors, lenses and windows. They provide a valuable tool at reasonable cost that may, relatively simply, be used to automate the alignment process.

\section{Acknowledgment}

This work was supported in part by the Swiss National Science Foundation.

\section{References}

[1] CRPP annual report (2005), p. 115

[2] Reusch, et al., Rev. Sci. Instrum. 79 (2008) 10 E733.

[3] Murmann, et al., Rev. Sci. Instrum. 74 (2003) 10.

[4] Makariou, et al., 19th IEEE/NPSS SOFT, 2002. 